Virginia Commonwealth University vCU Scholars Compass

2006

\title{
Magnetic and Mossbauer spectroscopy studies of nanocrystalline iron oxide aerogels
}

\author{
E. E. Carpenter \\ Virginia Commonwealth University, ecarpenter2@vcu.edu \\ J.W. Long \\ Naval Research Laboratory \\ D. R. Rolison \\ Naval Research Laboratory \\ See next page for additional authors
}

Follow this and additional works at: http://scholarscompass.vcu.edu/chem_pubs

Part of the Chemistry Commons

Carpenter, E. E., Long, J. W., Rolison, D. R., et al. Magnetic and Mössbauer spectroscopy studies of nanocrystalline iron oxide aerogels. Journal of Applied Physics 99, 08 N711 (2006). Copyright (C) 2006 AIP Publishing LLC.

\section{Downloaded from}

http://scholarscompass.vcu.edu/chem_pubs/39

This Article is brought to you for free and open access by the Dept. of Chemistry at VCU Scholars Compass. It has been accepted for inclusion in Chemistry Publications by an authorized administrator of VCU Scholars Compass. For more information, please contact libcompass@vcu.edu. 
Authors

E. E. Carpenter, J. W. Long, D. R. Rolison, M. S. Logan, K. Pettigrew, R. M. Stroud, L. Theil Kuhn, B. Rosendahl Hasen, and S. Mørup 


\title{
Magnetic and Mössbauer spectroscopy studies of nanocrystalline iron oxide aerogels
}

\author{
E. E. Carpenter ${ }^{\mathrm{a})}$ \\ Department of Chemistry, Virginia Commonwealth University, Richmond, Virginia 23284
}

J. W. Long, D. R. Rolison, M. S. Logan, K. Pettigrew, and R. M. Stroud

Naval Research Laboratory, Washington, DC 20375

L. Theil Kuhn

Materials Research Department, RisøNational Laboratory, Frederiksborgvej 399, DK-4000 Roskilde, Denmark

B. Rosendahl Hansen and S. Mørup

Department of Physics, Technical University of Denmark, Building 307, DK-2800 Kongens Lyngby, Denmark

(Presented on 2 November 2005; published online 28 April 2006)

\begin{abstract}
A sol-gel synthesis was used to produce iron oxide aerogels. These nanocrystalline aerogels have a pore-solid structure similar to silica aerogels but are composed entirely of iron oxides. Mössbauer experiments and x-ray diffraction showed that the as-prepared aerogel is an amorphous or poorly crystalline iron oxide, which crystallized as a partially oxidized magnetite during heating in argon. After further heat treatment in air, the nanocrystallites are fully converted to maghemite. The particles are superparamagnetic at high temperatures, but the magnetic properties are strongly influenced by magnetic interactions between the particles at lower temperatures. (C) 2006 American Institute of Physics. [DOI: 10.1063/1.2176894]
\end{abstract}

\section{INTRODUCTION}

Aerogels are promising platforms for a variety of applications due to their inherent characteristics of high surface areas and through-connected networks of mesopores, which facilitate the transport and interaction of liquid- or gas-phase agents within the aerogel structure. Magnetic iron oxides, such as $\gamma-\mathrm{Fe}_{2} \mathrm{O}_{3}$ (maghemite) or $\mathrm{Fe}_{3} \mathrm{O}_{4}$ (magnetite), have been incorporated into $\mathrm{SiO}_{2}$ aerogel matrices, combining the desirable structural characteristics of the aerogel with magnetic properties of the iron oxide. ${ }^{1-8}$ Recently, Long et al. ${ }^{6}$ adapted a highly flexible sol-gel method to prepare freestanding $\mathrm{FeOx}$ aerogels with the specific goal of generating magnetic aerogel nanoarchitectures. Controlled temperature/ atmosphere treatments were applied to convert the amorphous as-prepared $\mathrm{FeOx}$ aerogels into mixed-phase nanocrystalline $\mathrm{Fe}_{3} \mathrm{O}_{4} / \gamma-\mathrm{Fe}_{2} \mathrm{O}_{3}$ forms, with the specific crystalline phase being highly dependent on the atmosphere (inert versus oxidizing) used during the crystallization. All nanocrystalline $\mathrm{FeOx}$ aerogels exhibited characteristic superparamagnetic behavior, whereby the magnetic moments of the nanoparticles spontaneously are reversing due to thermal excitations while still retaining the desirable aerogel morphology.

In this report, we describe the magnetic and Mössbauer spectroscopy analyses of two forms of nanocrystalline $\mathrm{FeOx}$ aerogels. These characterizations provide additional insight into the crystal structure, oxidization state, and magnetic properties of the aerogels.

${ }^{a)}$ Electronic mail: ecarpenter2@vcu.edu

\section{EXPERIMENT}

Iron oxide aerogels were prepared by epoxide-driven sol-gel chemistry methods and subsequent supercritical $\mathrm{CO}_{2}$ extraction, as described previously. ${ }^{6}$ Thermal treatments were performed in either static ambient air or flowing argon (linear flow rate $=56 \mathrm{~cm} \mathrm{~min}^{-1}$ ) to convert the amorphous as-prepared $\mathrm{FeOx}$ aerogels to nanocrystalline forms. The various samples are labeled according to their thermal treatments: "260-ARG" denotes as-prepared $\mathrm{FeOx}$ aerogels heated under argon flow at $260{ }^{\circ} \mathrm{C}$ and "260-ARG-AIR" is used to denote the 260-ARG aerogel after an additional heat treatment at $260^{\circ} \mathrm{C}$ in static air.

Transmission electron microscopy was used to characterize the aerogel morphologies and to confirm particle size and crystal structure. Samples were prepared by dry grinding the aerogel powders and then brushing the dust onto holeycarbon film supports. The data were obtained using a JEOL 2010F transmission electron microscope (TEM) equipped with a Gatan charge coupled device (CCD) camera and Noran Vantage energy dispersive spectroscope (EDS). X-ray diffraction (XRD) was performed on ground powders of the $\mathrm{FeOx}$ aerogels using a Bruker D8 Advance $\mathrm{x}$-ray diffractometer. Scans were recorded for $2 \theta$ values between $20^{\circ}$ and $70^{\circ}$, using a step size of $0.02^{\circ}$ and integration of $16 \mathrm{~s} / \mathrm{step}$. For crystalline samples, the particle sizes were calculated from the $\mathrm{XRD}_{311}$ peak width using the Scherrer equation. A Quantum Design MPMS-5s superconducting quantum interference device (SQUID) magnetometer was used for magnetic characterization. The dried powder was placed in a gelatin capsule and the data were corrected for background due to the sample holder. Mössbauer spectra were obtained 

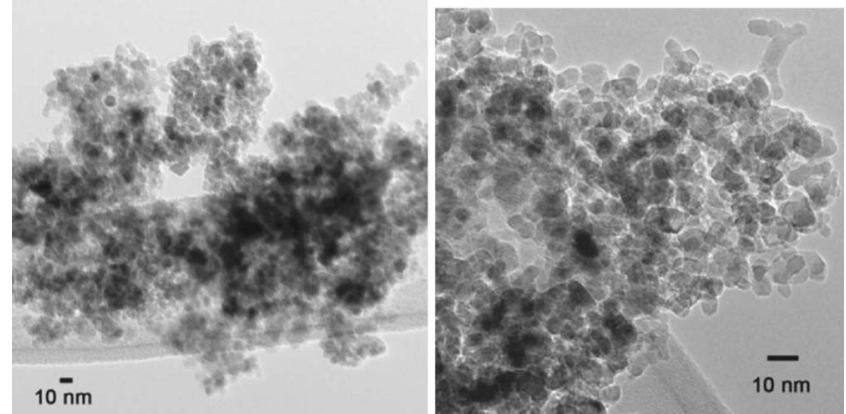

FIG. 1. Transmission electron microscopy of the 260-ARG (left) and 260ARG-AIR (right) aerogels.

using constant acceleration Mössbauer spectrometers with sources of ${ }^{57} \mathrm{Co}$ in rhodium. Low-temperature spectra were recorded by using a closed cycle helium refrigerator (APD Cryogenics Inc.). Spectra with applied magnetic fields were obtained using an iron-core electromagnet and the field was applied perpendicular to the $\gamma$ rays. Velocities are given relative to the centroid of the spectrum of a $12.5 \mu \mathrm{m} \alpha$-Fe foil, which was used for calibration.

\section{RESULTS AND DISCUSSION}

As demonstrated previously, ${ }^{6}$ a $260{ }^{\circ} \mathrm{C}$ heat treatment of the as-prepared $\mathrm{FeOx}$ aerogels under argon flow (sample 260-ARG) yields nanocrystalline structures with crystallite sizes of $\sim 7 \mathrm{~nm}$ [see Fig. 1(A)]. After further $260{ }^{\circ} \mathrm{C}$ heat treatment in air, the aerogel (sample 260-ARG-AIR) exhibits very similar morphology and crystallinity, with a slightly larger, $\sim 8.5 \mathrm{~nm}$, particle crystallite size [see Fig. 1(B)]. $\mathrm{X}$-ray diffraction data of the as-prepared sample did not show any significant diffraction peaks and therefore suggest that the sample was amorphous or poorly crystalline. The results of electron and $\mathrm{x}$-ray diffraction were inconclusive for both crystallized aerogel samples, as the data were consistent with both $\mathrm{Fe}_{3} \mathrm{O}_{4}$ and $\gamma-\mathrm{Fe}_{2} \mathrm{O}_{3}$ phases. Particle size estimated from TEM was difficult due to difficulty in isolating individual particles.

The Mössbauer spectrum of the as-prepared sample at $20 \mathrm{~K}$ (not shown) consists of a sextet with broad lines and a negligible quadrupole shift. The average magnetic hyperfine field is of the order of $45 \mathrm{~T}$. The parameters suggest, in accordance with the XRD results, that the sample consists of amorphous or poorly crystalline material, e.g., ferrihydrite. At $80 \mathrm{~K}$, the spectrum consists of a doublet with a quadrupole splitting of about $0.75 \mathrm{~mm} \mathrm{~s}^{-1}$. The collapse of the magnetic hyperfine splitting is presumably due to fast superparamagnetic relaxation. The Mössbauer spectra of the 260-ARG and 260-ARG-AIR aerogels in Figs. 2(a) and 2(b) show only magnetically split components at least up to $130 \mathrm{~K}$, whereas the room temperature spectra mainly consist of doublets because of fast superparamagnetic relaxation. The magnetic hyperfine field at $80 \mathrm{~K}$ was of the order of $48 \mathrm{~T}$, which is typical for nanoparticles of magnetite and maghemite. In the $130 \mathrm{~K}$ spectrum of the 260-ARG aerogel, line 1 is broader and less intense than line 6 . This asymmetry is qualitatively similar to, but smaller than that of, the spectrum of bulk magnetite above the Verwey temperature $(\sim 119 \mathrm{~K})$, and it
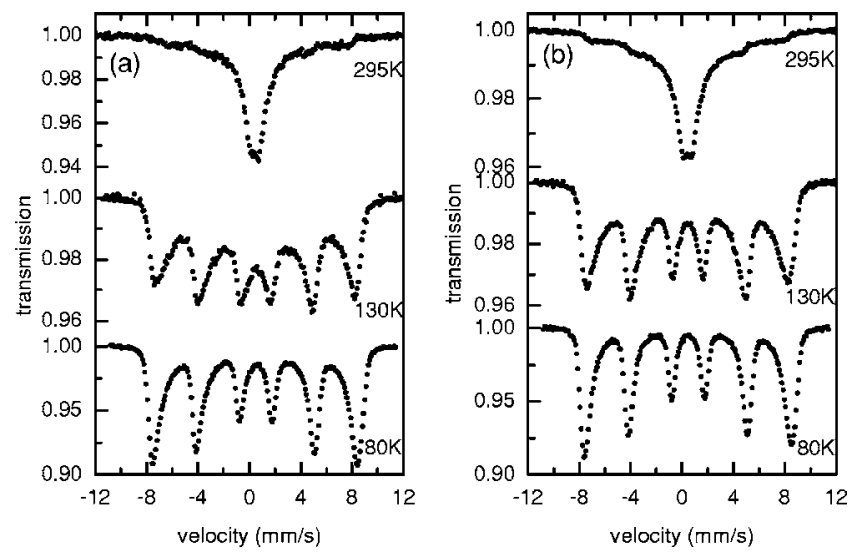

FIG. 2. Mössbauer spectra of aerogels (a) 260-ARG and (b) 260-ARG-AIR at the indicated temperatures.

indicates that the nanoparticles have a composition intermediate between that of magnetite and maghemite. ${ }^{9}$ The lowtemperature spectra of the 260-ARG-AIR aerogel show the opposite asymmetry and are typical for pure maghemite. ${ }^{10}$

Application of a magnetic field $B_{a}$ to the nanoparticles may block the superparamagnetic relaxation of the magnetic moments and provide us with a means to estimate the magnetic moment of the particles. ${ }^{7,8}$ Above the blocking temperature the induced magnetic hyperfine field, $B_{\text {ind }}=\mid \boldsymbol{B}_{\text {obs }}$ $-\boldsymbol{B}_{a}$, is related to the magnetic moment $\mu$ via $\boldsymbol{B}_{\text {obs }} \cong \boldsymbol{B}_{0}(1$ $\left.-k_{B} T / \mu B_{a}\right)+\boldsymbol{B}_{a}$. In the special cases of magnetite and maghemite, which are ferrimagnets, the relation can be written as $B_{\text {ind }}=B_{\text {obs }}+x B_{a}$, where $x$ is the fraction of excess ferrimagnetic spins (for maghemite $x \approx 1 / 4$ ). Figure 3(a) shows the spectra for the 260-ARG aerogel at 130 and $295 \mathrm{~K}$ with applied fields up to $1.5 \mathrm{~T}$, and it is clear that the magnetic field restores the magnetic splitting at room temperature as expected for superparamagnetic particles. By plotting the induced hyperfine field of sample 260-ARG-AIR at $295 \mathrm{~K}$ as a function of the inverse applied field and by fitting a straight line [Fig. 3(b)] we find $\mu \approx 1.2 \times 10^{-19} \mathrm{~A} \mathrm{~m}^{2}$. Using the relation $\mu=M V$, where $M$ is the magnetization and $V$ is the volume, and assuming that the magnetization of the nanoparticles in the 260-ARG-AIR aerogel is the same as for bulk $\gamma-\mathrm{Fe}_{2} \mathrm{O}_{3} \quad\left(M=4.3 \times 10^{5} \mathrm{~A} / \mathrm{m}\right)$, we find $V=2.8 \times 10^{-25} \mathrm{~m}^{3}$, i.e., an average diameter of $\sim 8 \mathrm{~nm}$, in good agreement with the TEM data.

The lines of the sextet in the zero-field spectra obtained
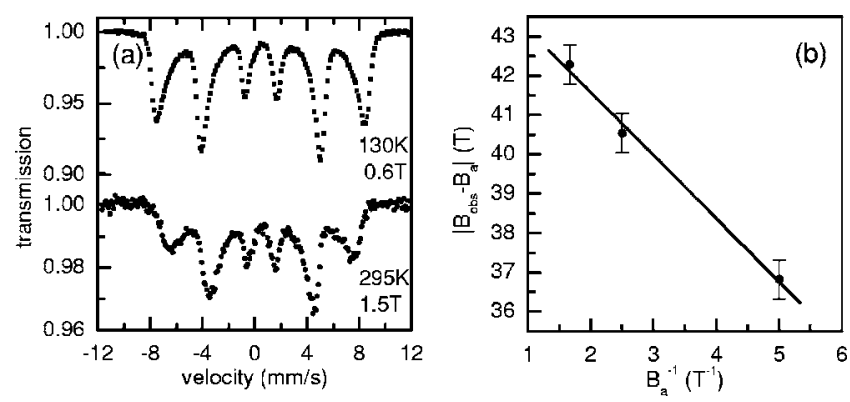

FIG. 3. (a) Mössbauer spectra of the 260-ARG aerogel at the indicated temperatures and applied fields. (b) Field dependence of the induced hyperfine field at $295 \mathrm{~K}$ used for the size estimation in sample 260-ARG-AIR. 

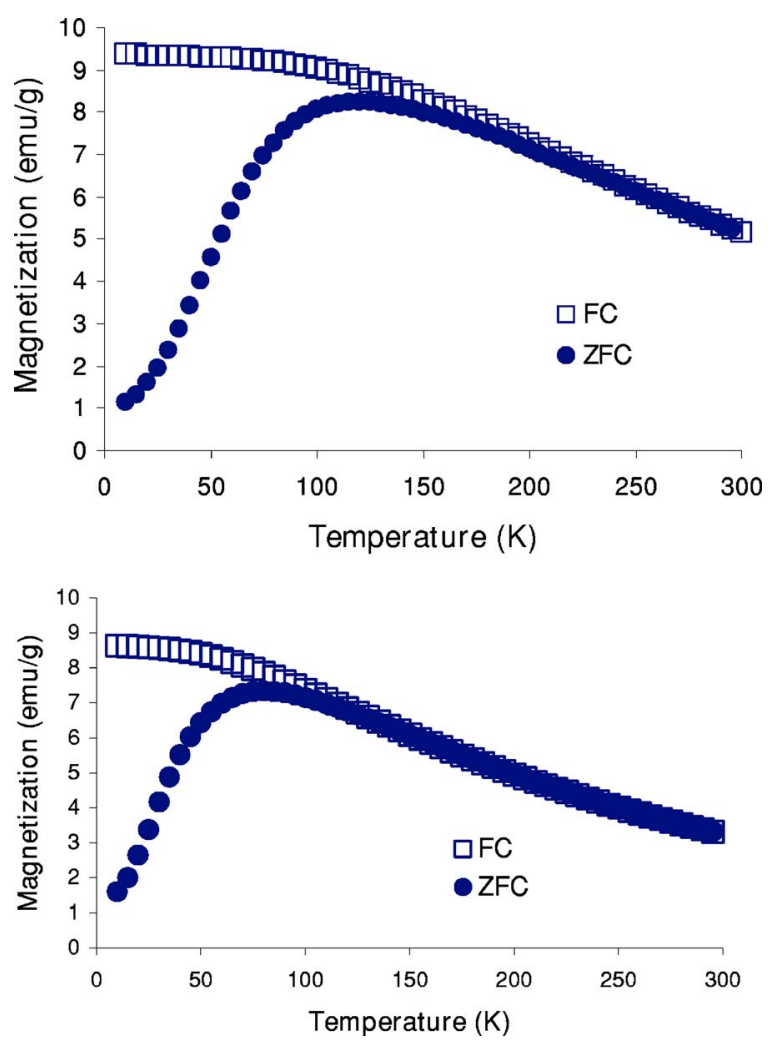

FIG. 4. (Color online) Magnetization vs temperature of 260-ARG-AIR (top) and 260-ARG (bottom). The data were obtained with an applied field of $0.01 \mathrm{~T}$.

at $130 \mathrm{~K}$ are very broad and there is no indication of a doublet due to particles with fast superparamagnetic relaxation, although maghemite particles with similar size, but suspended in a liquid, show spectra with an intense doublet at this temperature due to particles with fast superparamagnetic relaxation. ${ }^{8}$ This indicates that the magnetic properties of the aerogel samples are strongly influenced by magnetic interactions between the particles such that the superparamagnetic relaxation is suppressed. ${ }^{8,9}$ The magnetic splitting in the spectrum of the air-heated sample is better resolved than that of the argon-heated samples. This may be explained by an enhanced interaction in this sample and/or by an increase of the particle size.

Zero-field (ZFC) and field (FC) cooled magnetization curves were measured at applied fields of $0.01 \mathrm{~T}$. The data are presented in Fig. 4. Evidently, the peak in the ZFC curve is at a higher temperature for the 260-ARG-AIR sample than for the 260-ARG sample, 120 and $80 \mathrm{~K}$, respectively. The peak temperature is related to the blocking temperature of the superparamagnetic particles and depends on the particle size, the magnetic anisotropy, and the strength of interparticle interactions. The difference between the two samples can be explained by a stronger interparticle interaction and/or an increase of the particle volume during heating in air. The width of the ZFC curve also increases upon heating in air. This is presumably due to a broader distribution of the strength of interparticle interactions in sample 260-ARGAIR. For both samples, the FC curves flatten out at the lowest temperatures in a way typical for interacting particles. ${ }^{10}$ This is in contrast to the strong increase of the signal with decreasing temperature seen in typical samples of noninteracting particles. ${ }^{10}$ Thus the magnetization data give further evidence for the importance of magnetic interactions between the particles.

\section{CONCLUSIONS}

The results of x-ray diffraction and Mössbauer spectroscopy measurements show that the as-prepared aerogel is amorphous or poorly crystalline. After heating in argon, a spinel structure is formed, and the Mössbauer data indicate that it is an intermediate between magnetite and maghemite, i.e., a partially oxidized magnetite. When the sample subsequently was heated in air, it fully converted to maghemite with a slightly larger particle size. Both the Mössbauer and magnetization measurements show that the particles are superparamagnetic at high temperatures, but the magnetic properties at lower temperatures are strongly affected by magnetic interactions between the particles.

${ }^{1}$ C. Cannas et al., J. Mater. Chem. 11, 3180 (2001).

${ }^{2}$ L. Casas, A. Roig, E. Rodriguez, E. Molins, J. Tejada, and J. Sort, J. Non-Cryst. Solids 285, 37 (2001).

${ }^{3}$ L. I. Casas, A. Roig, E. Molins, J. M. Greneche, J. Asenjo, and J. Tejada, Appl. Phys. A: Mater. Sci. Process. 74, 591 (2002).

${ }^{4}$ M. F. Casula, A. Corrias, and G. Paschina, J. Non-Cryst. Solids 293, 25 (2001).

${ }^{5}$ P. Fabrizioli, T. Burgi, and A. Baiker, J. Catal. 206, 143 (2002).

${ }^{6}$ P. Fabrizioli, T. Burgi, M. Burgener, S. van Doorslaer, and A. Baiker, J. Mater. Chem. 12, 619 (2002).

${ }^{7}$ C. T. Wang and R. J. Willey, Catal. Today 52, 83 (1999).

${ }^{8}$ C. T. Wang and R. J. Willey, J. Catal. 202, 211 (2001).

${ }^{9}$ E. Schmidbauer and M. Keller, J. Magn. Magn. Mater. 297, 107 (2006).

${ }^{10}$ P. J. Pollard, Hyperfine Interact. 41, 509 (1988). 